\title{
Utilizing Stability Index Tracing for Precise Load Buses Identification in Load Shedding Problem
}

\author{
Z. Hamid ${ }^{1}$, I. Musirin' ${ }^{2}$, M. M. Othman ${ }^{3}$ \\ Faculty of Electrical Engineering, Universiti Teknologi MARA, Selangor, Malaysia \\ Email: zulcromok086@gmail.com ${ }^{1}$, ismailbm1@gmail.com², mamat505my@yahoo.com³
}

Received 2013

\begin{abstract}
This paper proposes a new approach for suitable load buses identification via stability index tracing in performing corrective load shedding. The proposed identification technique is called the Fast Voltage Stability Index Load Tracing (FVSI-LT). By implementing a power tracing algorithm, a group of major contributors on the stress experienced by a power system is able to be precisely identified by a system operator (SO) based on the traced values of FVSI. To be precise, the traced FVSI via FVSI-LT can be used to form a ranking list indicating the priority of buses committed for shedding purpose. After designing a Fuzzy Inference System (FIS) for deciding the allowable load powers to be shed and performing experiment on IEEE 57-Bus reliability test system (RTS), it is revealed that the ranking list provided by FVSI-LT results to the most consistent improvement in terms of voltage stability and losses minimization.
\end{abstract}

Keywords: fuzzy logic, FVSI-LT, load shedding, stability index tracing

\section{Introduction}

Adequacy of reactive power support plays a significant role in guarantying the health of a power system from any disturbances [1]. Sudden line and generators outages and extreme demand increases will cause reactive power insufficiency. As a result, the operating point of a power system moves very close to a critical point known as Saddle Note Bifurcation (SNB) of $Q-V$ curve. When such disturbances dominate, any further reduction on reactive power support will lead to the worst phenomenon; namely voltage collapse or 'black-out' [2]. Scheduling on generators output powers, sizing on capacitor banks and transformers tap settings, and power flow control via Flexible Alternating Current Transmission System (FACTS) devices are the instances for counteracting any instability problems. However if all of them are exhausted, the only way for system recovery is by performing load shedding. Article [3] - [5] reported the techniques for rehabilitating the power system from being dominated by voltage collapse via under voltage load shedding (UVLS).

Meanwhile, various electricity tracing theories or simply power tracing algorithms have been proposed for solving transmission service pricing problems. Article [6] is considered as the pioneered method for tracing algorithm. A circuit theory based power tracing has been proposed by [7]. A unique tracing algorithm was proposed by [8] without requiring any assumptions like PSP or matrix inversion process. Next, optimization approach for real power tracing was firstly demonstrated by [9]. Because of involving too difficult problem formulation (too many constraints to be considered), the technique is less preferred for solving deregulated market problems. An Artificial Intelligence (AI) based tracing algorithm was firstly presented by [10] - [11].

This paper proposes a new approach for suitable load buses identification using stability index tracing. Instead of tracing the magnitude of powers as what others did, the proposed technique traces the Fast Voltage Stability Index (FVSI) contributed by load buses for better accuracy using any tracing algorithms; hence it is named as FVSI-Load Tracing (FVSI-LT). From the traced FVSI, a ranking list indicating the priority of load buses to be shed can be formed by a system operator (SO) for precise load buses selection prior to load shedding.

\section{The Stability Index Tracing}

In this paper, the Fast Voltage Stability Index (FVSI) is selected for the purpose of stability index tracing [12] [13]. Since FVSI is a line based index (which means that it is only used to indicate stress experienced by a particular line), there is a need to modify the index for indicating load bus that contributes high stress on that line. This can be achieved by performing load tracing algorithm or specifically called FVSI-LT. The FVSI of an 
l-th line can be represented in (1).

$$
\mathrm{FVSI}_{l}=\frac{4 Z_{l}^{2} Q_{r}}{V_{s}^{2} X_{l}}
$$

For a stable power system, the value of FVSI shall not exceed unity. Otherwise, the voltage collapse phenomenon will dominate.

\subsection{FVSI Modification for FVSI-LT}

Load tracing is defined as a task to trace the powers (generators power, line flows, and losses) extracted by an individual load. Due to such fact, tracing the stability index is termed FVSI-Load Tracing (FVSI-LT). According to [9], a power to be traced can be expressed as a summation of individual load participation in that power. Thus, an l-th line FVSI can also be expressed as a summation of individual load participation, as in (2).

$$
F V S I_{l}=F V S I_{l}^{1}+F V S I_{l}^{2}+\ldots+F V S I_{l}^{i, \text { nload }}
$$

Substituting (1) into (2) in the context of load tracing:

$$
\begin{gathered}
F V S I_{l}=\frac{4 Z_{l}^{2} Q_{r}^{1}}{V_{s}^{2} X_{l}}+\frac{4 Z_{l}^{2} Q_{r}^{2}}{V_{s}^{2} X_{l}}+\ldots+\frac{4 Z_{l}^{2} Q_{r}^{i, \text { nload }}}{V_{s}^{2} X_{l}} \\
F V S I_{l}=\frac{4 Z_{l}^{2}}{V_{s}^{2} X_{l}}\left(Q_{r}^{1}+Q_{r}^{2}+\ldots+Q_{r}^{i, \text { nload }}\right) \\
\therefore F V S I_{l}=\frac{4 Z_{l}^{2}}{V_{s}^{2} X_{l}} \sum_{i=1}^{\text {nload }} Q_{r}^{i}
\end{gathered}
$$

According to [9], the participation of a load with power $Q_{L i}$ in receiving end line flow can be expressed as in (6).

$$
Q_{r}^{i}=x_{r}^{i} \cdot Q_{L i}
$$

Thus, substituting (6) into (5):

$$
\therefore F V S I_{l}=\frac{4 Z_{l}^{2}}{V_{s}^{2} X_{l}} \sum_{i=1}^{\text {nload }} x_{r}^{i} \cdot Q_{L i}
$$

It can be deduced that from (7), the FVSI of $l$-th line contributed by $i$-th load of power $Q_{L i}$ can be mathematically represented as in (8).

$$
F V S I_{l}^{i}=\frac{4 Z_{l}^{2}}{V_{s}^{2} X_{l}}\left(x_{r}^{i} \cdot Q_{L i}\right)
$$

Thus, the only way for tracing FVSI contributed by a load bus is by tracing the receiving end power fraction, $x_{r}^{i}$ via any power tracing algorithms, as proposed in [6], [11] and [14]. It is recommended to explore article [15] for a brief explanation on how to perform FVSI-LT using the existing tracing algorithms. By determining the traced FVSI as in (8), the system operator (SO) is able to make a ranking list of load buses precisely indicating their priority to be shed.

\subsection{Application of FVSI-LT}

This section demonstrates the method for ranking load buses according to their priority based on the traced FVSI. Consider an IEEE 6-Bus reliability test system (RTS) as in Figure 1 with the calculated FVSI on line between bus 3 and $4\left(\mathrm{FVSI}_{3-4}\right)$, bus 4 and $6\left(\mathrm{FVSI}_{6-4}\right)$, and bus 5 and $6\left(F_{V S I}{ }_{6-5}\right)$. After performing FVSI-LT, the traced FVSI values contributed by each load are also included in Figure 1. For example, FVSI on line between bus 4 and 6 contributed by load at bus 6 is noted as $F_{V S I}-4(\mathrm{~L} 6)$. From these values, a ranking list is tabulated as in Table 1.

Table 1. Bus and Line Ranking Based on FVSI-LT

\begin{tabular}{cllc}
\hline \hline Rank & Lines & Buses & Traced FVSI \\
\hline 1 & $6-4$ & 3 & 0.50 \\
2 & $6-5$ & 3 & 0.40 \\
3 & $3-4$ & 3 & 0.30 \\
4 & $6-4$ & 5 & 0.25 \\
5 & $6-4,6-5,3-4$ & 4,5 & 0.20 \\
6 & $3-4,6-5,6-5,3-4$ & $4,5,6$ & 0.10 \\
7 & $6-4$ & 6 & 0.05 \\
\hline \hline
\end{tabular}

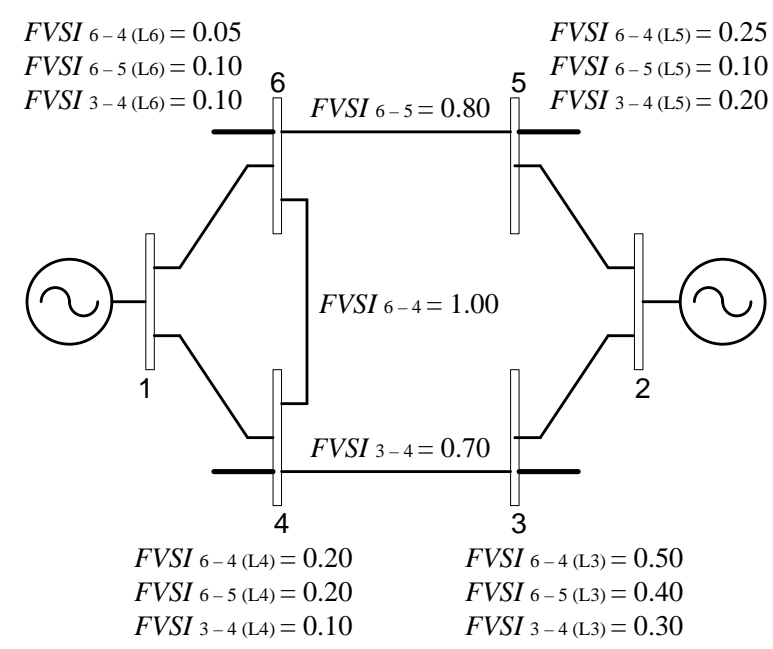

Figure 1. IEEE 6-bus system with single line outage

From Table 1, bus 3 possesses the highest traced FVSI and followed by bus 5, 4, and 6. From this example, bus 3 becomes the major contributor for the stress experienced 
by transmission line between bus 4 and 6 . The SO has to perform load shedding by prioritizing bus 3 as the first location to be shed, followed by bus 5,4 , and lastly bus 6 .

\section{Problem Formulation via Fuzzy System}

The purpose of performing FVSI-LT is to create a ranking list for precise load buses identification committed for shedding purpose. However, deciding suitable amount of load powers to be shed is the most critical task in load shedding problem as this will not only affect the post shedding condition, but also economical factors. Too many load buses interrupted during load shedding is not very effective. Hence, for fair amount of load powers decided for shedding with satisfactory voltage stability improvement, a decision making system has to be implemented, namely Fuzzy Inference System (FIS). For the purpose of this paper, only intuitive

\subsection{Fuzzy Logic Concept}

At first, it was proposed as a fuzzy set theory by L. A. Zadeh in 1965 . Contrary to binary logic that takes only two values (either 0 or 1 ), designing a system via fuzzy logic can have the value in between; that is the mapped value from a crisp value is between 0 and 1 . This enhances the flexibility of a FIS to be a good decision maker besides requiring only simple problem formulation. In deciding a crisp output value, a FIS infers one or more inputs based on fuzzy decision rule or if-then rule. There are two parts in the rule which are antecedent (input parts) and consequent (output part). The crisp values of input and output are mapped between 0 and 1 using membership functions; a group of linguistic variables plotted in graph form. A fuzzy rule consists of two-input antecedent and one output consequent is as follows.

$$
R_{i} \text { : IF } x_{1} \text { IS A AND } x_{2} \text { IS } B \text {, THEN } y \text { IS } C
$$

Where, $R_{i}$ is the $i$-th rule, $x_{1}$ and $x_{2}$ are the inputs, $y$ is the output, $A, B$, and $C$ are the linguistic values specified within the input and output space. The basic decision making process of a FIS is divided to five steps; (1) fuzzify input; (2) apply fuzzy operator; (3) apply implication method; (4) aggregate all outputs; (5) defuzzification [16].

\subsection{Proposed Load Shedding Scheme}

There are various techniques implemented for designing membership functions of FIS. Intuition, rank ordering, and probabilistic approach (optimization technique) [17] are the examples of techniques utilized for better membership functions design. Due to simplicity during problem formulation, intuitive technique is preferable. In this paper, the inputs to the designed FIS are the maximum
FVSI $\left(F V S I_{\max }\right)$ and minimum voltage magnitude $\left(V_{\min }\right)$ of the system, whereas the output is the percentage of allowable load powers to be shed (SL). The fuzzy rules are constructed based on the decisions as tabulated in Table 2.

Table 2. Fuzzy decision table

\begin{tabular}{|c|c|c|c|c|c|c|c|c|}
\hline & \multicolumn{7}{|c|}{$V_{\text {min }}$} \\
\hline & & EL & VL & $\mathrm{L}$ & M & $\mathrm{S}$ & VS & ES \\
\hline \multirow{7}{*}{$F V S I_{\max }$} & VL & $\mathrm{L}$ & $\mathrm{L}$ & $\mathrm{L}$ & VL & $\mathrm{VL}$ & EL & EL \\
\hline & $\mathrm{L}$ & M & M & $\mathrm{L}$ & $\mathrm{L}$ & VL & VL & EL \\
\hline & M & $\mathrm{H}$ & M & M & M & $\mathrm{L}$ & $\mathrm{L}$ & VL \\
\hline & $\mathrm{H}$ & $\mathrm{H}$ & $\mathrm{H}$ & M & M & M & $\mathrm{L}$ & $\mathrm{L}$ \\
\hline & $\mathrm{VH}$ & VH & $\mathrm{H}$ & $\mathrm{H}$ & $\mathrm{H}$ & M & $\mathrm{M}$ & $\mathrm{L}$ \\
\hline & S & $\mathrm{EH}$ & VH & VH & VH & $\mathrm{H}$ & $\mathrm{H}$ & $\mathrm{M}$ \\
\hline & VS & $\mathrm{EH}$ & $\mathrm{EH}$ & $\mathrm{EH}$ & VH & VH & $\mathrm{H}$ & $\mathrm{H}$ \\
\hline
\end{tabular}

In addition, there are seven linguistic variables for each input and output, as follows.

- $F V S I_{\max }$ : very low (VL), low (L), medium (M), high $(\mathrm{H})$, very high $(\mathrm{VH})$, stress $(\mathrm{S})$, very stress (VS).

- $V_{\text {min }}$ : extremely low (EL), very low (VL), low (L), medium (M), stable (S), very stable (VS), extremely stable (ES).

- SL: extremely low (EL), very low (VL), low (L), medium $(\mathrm{M})$, high $(\mathrm{H})$, very high $(\mathrm{VH})$, extremely high (EH).

Based on heuristic approach, seven linguistic variables were selected for better output. In addition, the trapezoidal and triangular membership functions were selected due to good sensitivity on to the change in input values. As reported in [17] - [18], the maximum allowable percentage of load power to be shed is 60 percents to 80 percents.
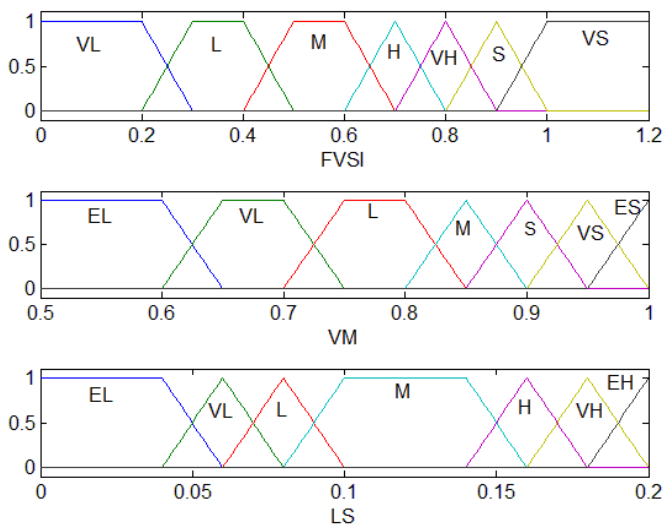

Figure 2. Proposed membership functions for FIS

Since the proposed load shedding scheme is performed iteratively (stage-by-stage), it is better to limit the percentage of shed load for each stage to a certain value. For the purpose of this paper, 20 percents (or 0.2) of shed load (SL) per stage is used and it is for both real and reactive load power. The proposed membership functions for the designed FIS are depicted in Figure 2. For the 
algorithm, the iterative strategy for performing load shedding via FIS is illustrated in Figure 3.

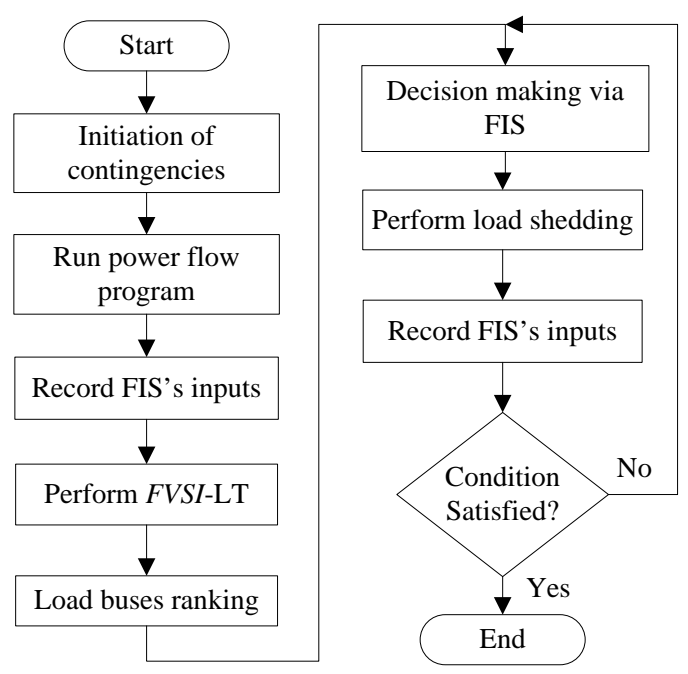

Figure 3. Load shedding strategy via IFIS

The algorithm starts by initiating disturbances to a test system. A power flow program is simulated to obtain the stability condition at pre load shedding. Thus, the resulted $F V S I_{\max }$ and $V_{\min }$ are recorded and will be assigned as the inputs to fuzzy system. Later, a power tracing algorithm is implemented to perform FVSI-LT and the traced FVSI values are used for ranking load buses according to their priority. At this stage, an iterative load shedding begins by feeding inputs to the designed FIS for determining suitable amount of shed load powers, SL. Next, $N_{\text {shed }}$ (number of loads) loads are selected based on the created ranking list; that is, the topmost load bus is firstly selected prior to selecting the next load bus. Then, the power of selected load bus is shed based on FIS output (SL values) according to (9).

$$
S_{\text {shed }}=P_{\text {shed }}+j Q_{\text {shed }}=S L .\left(P_{D}+j Q_{D}\right)
$$

After that, the condition of test system is evaluated via power flow program and the resulted $F V S I_{\max }$ and $V_{\text {min }}$ are recorded. If the stability condition at post shedding is still unsatisfactory, similar looping process as illustrated in Figure 3 is required. Otherwise, the overall algorithm is terminated.

\section{Results and Discussion}

The proposed technique was implemented using MATLAB software and validated on IEEE 57-Bus RTS. For this paper, there are five ranking methods for load buses identification before performing load shedding. Two of them are based on FVSI-LT with different tracing algo- rithms. Whereas the remaining three (non FVSI-LT) are Loss Sensitivity (LS) [19], Risk of Voltage Instability Index (RVI) [17], and Voltage Magnitude based ranking (VM) [20]. The first FVSI-LT is marked as FVSI-LT $\mathrm{L}_{\mathrm{A}}$ in which the tracing algorithm is based on optimization technique as proposed in [15]. The second one is FVSI-LT $\mathrm{B}_{\mathrm{B}}$ with TGLDF [6] as the tracing algorithm. In conducting the experiment, all ranking methods are analyzed under four levels of contingencies (marked as CL) as follows.

- $\quad$ CL1: Line outage: line 11, 15, 24, 51, 59, 75

- CL2: Generator outage: bus 2, 3, 6, 9

- CL3: Line outage: line 11, 15, 24, 51, 59, 75 Generator outage: bus 2, 3, 6, 9

- CL4: Line outage: line 11, 15, 24, 51, 59, 75 Generator outage: bus 2, 3

Load increase: $5 \%$ of total load power

The result details for post load shedding in terms of $V_{\min }, F V S I_{\max }$, and losses $P_{\text {loss }}$ are tabulated in Appendix, Table A.

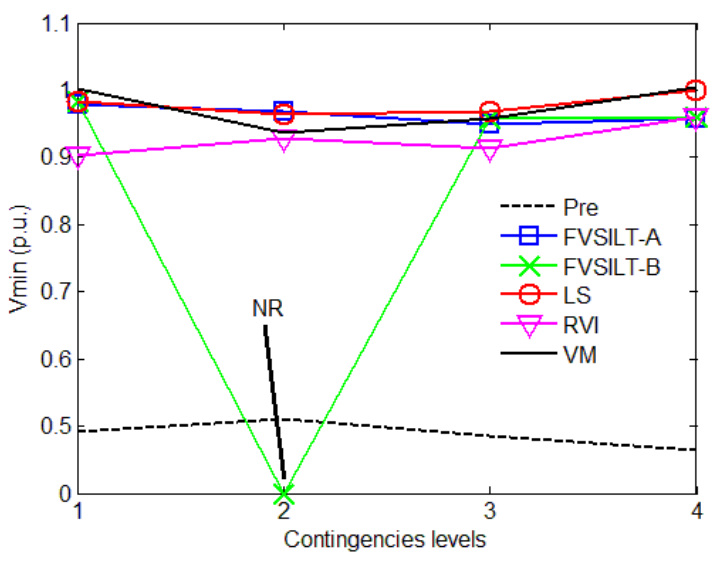

Figure 4. $V_{\min }$ trend at post load shedding

In the table, the pre load shedding condition (marked as 'pre') means the condition before load shedding was performed. From Table $\mathbf{A}$, the graphical illustrations concerning $V_{\min }, F V S I_{\max }$, and $P_{\text {loss }}$ are depicted in Figure 4, Figure 5, and Figure 6 respectively. Firstly, by looking at Figure $\mathbf{4}$ all methods provide significant improvement on voltage profile throughout the contingencies levels. As compared to pre condition, $V_{\min }$ has been improved to satisfactory magnitude of above than 0.90 p.u. It is seen that $F V S I-\mathrm{LT}_{\mathrm{A}}$, LS, and VM provide optimal improvement on $V_{\min }$ with magnitude of approximately 1.00 p.u. for all levels. The resulted trend of $F_{V S I}$ max at post load shedding is depicted in Figure 5. This time, both FVSI-LT $\mathrm{A}_{\mathrm{A}}$ and FVSI-LT $\mathrm{C}_{\mathrm{B}}$ are the best ranking methods with the lowest trend of $F V S I_{\max }$ through all contingencies levels (below than 0.20). The conventional ranking methods (LS, RVI, and VM) result to unstable trend of reduction as there exists fluctuation 
at CL1 and CL2 with magnitude of FVSI $I_{\max }$ of above than 0.20 .

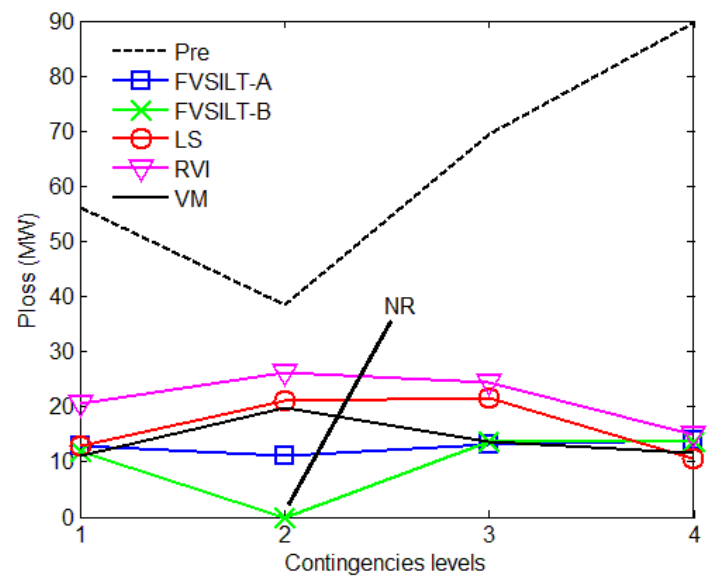

Figure 6. $P_{\text {loss }}$ trend at post load shedding

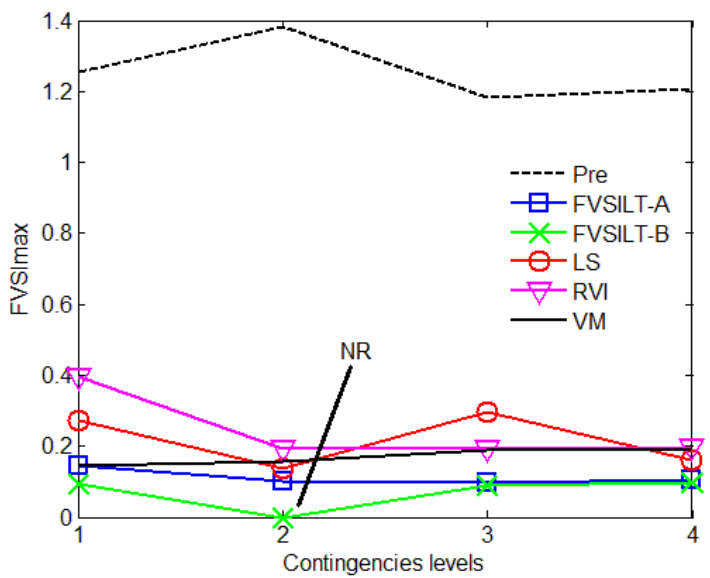

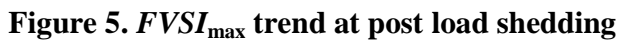

Similar trend as $F V S I_{\max }$ is observed for losses trend in Figure 6. The most consistent improvement on $P_{\text {loss }}$ is resulted by both FVSI-LT methods with reduced losses of about $10 \mathrm{MW}$, however there is still no result at CL2 for $F V S I-L_{\mathrm{B}}$. Unstable trend of losses reduction is depicted by conventional ranking methods through all levels; implying that they are not very consistent as FVSI-LT. Moreover, the reduced $P_{\text {loss }}$ is not as good as the proposed technique especially at CL2 and CL3. From all analysis, it is revealed that the most consistent improvement can only be provided via FVSI-LT $\mathrm{A}_{\mathrm{A}}$. Based on Figure 4 to Figure 6, the improvement trend resulted by this method is stable and optimal regardless of contingencies severity. As can be seen in Figure 5 and Figure 6, their improvement trend consists of large fluctuation at CL1 to CL3 and the resulted FVSI $I_{\max }$ and $P_{\text {loss }}$ at those levels is not as good as FVSI-LT $\mathrm{A}_{\mathrm{A}}$. Moreover in providing satisfactory voltage stability improvement, the number of loads involved for load shedding, $N_{\text {shed }}$ required by conventional ranking methods are higher than that of
FVSI-LT. From Table A, VM is the only method that requires high $N_{\text {shed }}$ at all contingencies levels, followed by LS at CL2 and CL4, and RVI at CL3. Too many loads involved for shedding means large interruption occurs at consumer sites and this is not good for an efficient load shedding scheme.

\section{Conclusion}

In brief, a new approach for precise load buses identification has been proposed. The technique applied stability index tracing namely FVSI-LT for creating a useful ranking list of load buses. Based on the traced FVSI, the load buses are ranked according to their priority and this will help the system operator (SO) to perform accurate load buses selection prior to performing any corrective actions against voltage instability. After validating the reliability of ranking list in load shedding problem, it was justified that FVSI-LT is reliable as the improvement on voltage stability condition at post load shedding is consistent regardless of contingencies severity. As compared to other methods, ranking list via FVSI-LT provides satisfactory improvement in terms of voltage magnitude, line stress, and losses minimization. Such discovery has proven the capability of power tracing to be an alternative for voltage stability improvement besides solving power system economics problems as what other researches proposed.

\section{Acknowledgement}

The authors would like to acknowledge The Research Management Institute (RMI) UiTM, Shah Alam, Human Resource Department of UiTM and Ministry of Higher Education Malaysia (MOHE) for the financial support of this research. This research is jointly supported by Research Management Institute (RMI) via the Excellence Research Grant Scheme UiTM with project code: 600-RMI/ST/DANA 5/3/Dst (164/2011) and MOHE under the Exploratory Research Grant Scheme (ERGS) with project code: 600-RMI/ERGS 5/3 (14/2011).

\section{REFERENCES}

[1] D. Devaraj , J. P. Roselyn, "Genetic Algorithm Based Reactive Power Dispatch for Voltage Stability Improvement”, Electrical Power And Energy Systems, Vol. 32, 2010, pp. 1151 - 1156.

[2] P. Pinceti, "Emergency Load-Shedding Algorithm for Large Industrial Plants”, Control Engineering Practice, Vol. 10, 2002, pp. $175-181$.

[3] F. M. Echavarren, E. Lobato, L. Rouco, “A Corrective Load Shedding Scheme to Mitigate Voltage Collapse”, International Journal of Electrical Power and Energy Systems, Vol. 28, 2006, pp. $58-64$.

[4] L. D. Arya, V. S. Pande, D. P. Kothari, “A Technique for Load-Shedding Based On Voltage Stability Consideration”, International Journal of Electrical Power and Energy Systems, Vol. 27, 2005, pp. 506 - 517. 
[5] T. Amraee, A. M. Ranjbar, B. Mozafari, N. Sadati, “An Enhanced Under-Voltage Load-Shedding Scheme to Provide Voltage Stability”, Electric Power Systems Research, Vol. 77, 2007, pp. $1038-1046$.

[6] J. Bialek, "Tracing the Flow of Electricity", in Generation, Transmission and Distribution, IEE Proceedings, Vol. 143, no. 4, July 1996, pp. 313 - 320.

[7] J. H. Teng, "Power Flow and Loss Allocation for Deregulated Transmission Systems", International Journal of Electrical Power and Energy Systems, Vol. 27, 2005, pp. 327-333.

[8] S.Abdelkader, "A Method for Determining Generators' Shares in Loads, Line Flows and Losses," Journal of the Franklin Institute, Vol. 344, 2007, pp. 1063-1074

[9] A. R. Abhyankar, S. A. Soman, S. A. Khaparde, "Optimization Approach to Real Power Tracing: An Application to Transmission Fixed Cost Allocation”, IEEE Transactions on Power Systems, Vol. 21, no. 3, August 2006, pp. 1350 - 1361.

[10] M. H. Sulaiman, M. W. Mustafa, H. Shareef, S. N. A. Khalid, O. Aliman, "Real and Reactive Power Flow Allocation in Deregulated Power System Utilizing Genetic-Support Vector Machine Technique", International Review of Electrical Engineering (IREE), Vol. 5, 2010, pp. $2199-2208$.

[11] M. H. Sulaiman, M. W. Mustafa, H. Shareef, S. N. A. Khalid, "An Application Of Artificial Bee Colony Algorithm with Least Squares Support Vector Machine for Real And Reactive Power Tracing in Deregulated Power System”, International Journal of Electrical Power and Energy Systems, Vol. 37, 2012, pp. 67 77.

[12] I. Musirin, T. K. A. Rahman, "Estimation of Maximum Loadability in Power Systems by Using Fast Voltage Stability
Index”, International Journal of Power and Energy Systems, Vol. 25, n. 3, 2005, pp. $181-189$.

[13] I. Musirin, T. K. A. Rahman, "Evolutionary Programming Based Optimization Technique for Maximum Loadability Estimation in Electric Power System", in National Proceedings of Power Engineering Conference (PECon) Dec. 2003. pp. 205 - 210.

[14] Z. Hamid, I. Musirin, M. M. Othman, M. N. A. Rahim, "New Formulation Technique for Generation Tracing via Evolutionary Programming”, International Review of Electrical Engineering (IREE), Vol. 6, 2011, pp. 1946 - 1959.

[15] Z. Hamid, I. Musirin, M. N. A. Rahim, N. A. M. Kamari, “Application of Electricity Tracing Theory and Hybrid Ant Colony Algorithm for Ranking Bus Priority in Power System”, International Journal of Electrical Power and Energy Systems, Vol. 43, 2012, pp. $1427-1434$

[16] T. J. Ross, Fuzzy Logic with Engineering Applications. John Wiley and Sons, 2009.

[17] J. Sasikala, M. Ramaswamy, "Fuzzy Based Load Shedding Strategies for Avoiding Voltage Collapse", Applied Soft Computing, Vol. 11, 2011, pp. 3179 - 3185.

[18] D. Mingchui, L. Chinwang, W. Chikong, "Adaptive Under-Frequency Load Shedding”, Tsinghua Science and Technology, Vol. 13, 2008, pp. $823-828$.

[19] J. Zhu, Optimization of Power System Operation: John Wiley and Sons, 2009

[20] A. M. A. Haidar, A. Mohamed, A. Hussain, "Vulnerability Control of Large Scale Interconnected Power System Using Neuro-Fuzzy Load Shedding Approach”, Expert Systems with Applications, Vol. 37, 2010, pp. 3171 - 3176.

\begin{tabular}{|c|c|c|c|c|c|c|}
\hline CL & Method & $N_{\text {shed }}$ & Top 10 load buses (based on priority) & $\begin{array}{c}V_{\min } \\
\text { (p.u.) }\end{array}$ & $F V S I_{\text {max }}$ & $\begin{array}{c}P_{\text {loss }} \\
(\mathrm{MW})\end{array}$ \\
\hline \multirow{6}{*}{1} & Pre & & . & 0.492 & 1.253 & 56.213 \\
\hline & $F V S I-\mathrm{LT}_{\mathrm{A}}$ & 16 & $30,25,31,35,38,47,49,28,15,18$ & 0.979 & 0.146 & 12.921 \\
\hline & FVSI-LT $\mathrm{B}_{\mathrm{B}}$ & 16 & $30,31,50,25,42,49,9,41,47,18$ & 0.984 & 0.095 & 11.852 \\
\hline & LS & 18 & $31,30,25,33,32,23,20,35,57,19$ & 0.983 & 0.274 & 12.848 \\
\hline & RVI & 23 & $8,12,3,6,9,1,2,17,18,19$ & 0.902 & 0.395 & 20.596 \\
\hline & VM & 29 & $30,31,25,33,32,23,35,57,20,56$ & 1.003 & 0.146 & 11.051 \\
\hline \multirow{6}{*}{2} & Pre & & - & 0.510 & 1.381 & 38.451 \\
\hline & $F V S I-\mathrm{LT}_{\mathrm{A}}$ & 19 & $30,25,38,50,2,31,35,53,14,3$ & 0.968 & 0.102 & 11.007 \\
\hline & $F V S I-\mathrm{LT}_{\mathrm{B}}$ & - & - & - & - & - \\
\hline & LS & 30 & $31,30,25,33,32,35,57,56,23,42$ & 0.963 & 0.139 & 21.087 \\
\hline & RVI & 22 & $8,1,12,2,13,17,3,5,6,14$ & 0.929 & 0.192 & 26.171 \\
\hline & VM & 31 & $30,31,25,33,32,35,57,56,23,42$ & 0.936 & 0.158 & 19.857 \\
\hline \multirow{6}{*}{3} & Pre & & - & 0.485 & 1.184 & 69.528 \\
\hline & $F V S I-\mathrm{LT}_{\mathrm{A}}$ & 15 & $31,30,25,49,9,2,3,14,35,38$ & 0.950 & 0.098 & 13.211 \\
\hline & $F V S I-\mathrm{LT}_{\mathrm{B}}$ & 15 & $30,31,50,42,25,2,49,3,47,41$ & 0.957 & 0.091 & 13.723 \\
\hline & LS & 16 & $31,30,25,33,32,23,20,35,57,19$ & 0.969 & 0.298 & 21.599 \\
\hline & RVI & 30 & $8,12,1,17,3,5,6,13,14,15$ & 0.914 & 0.192 & 24.459 \\
\hline & VM & 32 & $30,31,25,33,32,23,35,57,20,56$ & 0.957 & 0.189 & 13.758 \\
\hline \multirow{6}{*}{4} & Pre & & - & 0.464 & 1.209 & 89.738 \\
\hline & $F V S I-\mathrm{LT}_{\mathrm{A}}$ & 20 & $30,25,2,31,3,14,38,49,15,28$ & 0.957 & 0.107 & 13.955 \\
\hline & $F V S I-\mathrm{LT}_{\mathrm{B}}$ & 21 & $30,31,50,42,25,2,49,9,3,47$ & 0.959 & 0.098 & 13.671 \\
\hline & LS & 30 & $31,30,25,33,32,23,20,35,57,19$ & 1.000 & 0.163 & 10.701 \\
\hline & RVI & 20 & $8,12,6,9,1,17,3,15,16,18$ & 0.959 & 0.198 & 15.035 \\
\hline & VM & 33 & $31,30,25,33,32,35,23,57,20,56$ & 1.005 & 0.187 & 11.587 \\
\hline
\end{tabular}

